\title{
Browning in Apple Juice Processing and Its Control Technology
}

\author{
Yang Jing ${ }^{1}$, Wang Zihua ${ }^{2}$ \\ ${ }^{1}$ Jiangxi Vocational Technical College of Industrial Trade, Nanchang, 330038, China; \\ ${ }^{2}$ Nanchang Institute of Science \& Technology, Nanchang, 330108, China
}

Keywords: apple juice processing; browning; high temperature treatment; concentration ratio; inhibitor

\begin{abstract}
There are abundant soluble cellulose and vitamin C in apple juice, which is a healthy and healthy drink. In recent years, the consumption in China has increased greatly, and it has a broad consumption prospect. However, during the processing of apple juice, browning is easy to occur due to the existence of polyphenol oxidase, which reduces its nutritional value and sensory effects. This study studies the causes of the phenomenon and the inhibition technology, and compares the inhibition effect of different concentration inhibitors. The optimum ratio of the compound inhibitor is obtained by orthogonal test. It has important reference significance for the improvement of the processing technology of apple juice.
\end{abstract}

\section{Introduction}

China is a big apple producing country in the world. Apple has a large output in China, and is also loved by consumers. But in our country, there are few fresh fruit juice stores in our country at present. The main reason is that the polyphenol oxidase in fresh apple juice is easy to browning, and it is more troublesome to treat it. Compared with other fruits and vegetables, the enzymatic browning of apple in processing is particularly serious, and the research on its processing theory and technology has become one of the problems to be solved in the processing of apple after the processing. According to this, this study intends to study different methods of browning inhibition, by summarizing the effects of different methods, in order to get a better effect of composite inhibitors, and compare it with physical methods to reduce the browning in the processing of apple juice.

\section{The present situation and development prospect of 1 fresh apple juice at home and abroad}

With the continuous improvement of people's living standards, more and more people pay more attention to health care. The apple contains rich vitamin $\mathrm{C}$, which not only increases the taste of the fruit juice, but also has the convenience of drinking and is favored by the consumers. The development and prospects of the fresh apple juice are introduced.

\subsection{The present situation of domestic development of fresh apple juice.}

There are many kinds of fresh apple juice in China, including watermelon, orange juice, Hami melon and apple juice. Different kinds of fruit juices are sold in different seasons in the catering market. The fruit of fresh juice is derived from various regions of China, and the prices are different with the different seasons. However, at any time, Apple has the characteristics of easy storage, so it is also the main force of the consumption of fresh juice in the catering market. The catering enterprises can get the goods from the local fruit wholesale market. With the increasing demand, the domestic fresh juice market industry has developed rapidly, which has obtained greater benefits and juice processing enterprises. The industry has also continuously upgraded the technology, thus improving the quality of fresh juice. Therefore, the consumption potential of fresh juice in China is large, and it has a larger development space. 


\subsection{The present situation of foreign development of fresh apple juice.}

In foreign countries, the consumption market of fresh juice is obviously less than that of China. The sale of fresh juice is often sold in combination with other fruit and vegetable juices, which is more suitable for the middle-aged and the elderly, and the market attraction of the separate fresh juice is less. However, with the continuous improvement of people's living standards and the progress of science and technology, three high crowds are increasing gradually. People begin to realize the importance of nutrition and diet. Fresh apple juice has gradually been recognized by people, and the consumption market is gradually expanding.

\subsection{The development prospect of fresh apple juice.}

The fresh fruit juice is rich in nutrition, and can be collocated with many kinds of fruit to become a better taste drink. According to the experts of the food industry, the future apple juice fresh squeeze industry will rise and become the healthy drink of the popular food and beverage. However, browning will occur during the processing of apple juice, which seriously affects the nutritional value and sensory effects of fruit juice. Therefore, in order to avoid this problem, we need to add certain inhibitors to the processing of fruit juice, improve the color of the juice, let the juice do not browning in a short time, in order to increase the consumer's desire to buy, and develop more apple juice market.

\section{The browning in apple juice processing and its inhibition technology}

There is a kind of enzyme called polyphenol oxidase in apple, which is derived from phenolics, which can produce adjacent quinones, and the o-quinone itself can be polymerized by itself, and can also form a polymer complex with protein or amino acid, resulting in the formation of brown pigment. The formation of brown pigment affects the taste of apple juice. And appearance, will also cause the loss of fruit juice nutrients, or even deterioration. Therefore, in the process of apple juice production, according to the characteristics of processing raw materials, reasonable selection of browning inhibitor can improve the quality of the production of fruit juice, avoid the flow of nutrients and make the juice have a better appearance and taste.

$\mathrm{NaCl}$ browning inhibitor. Before apple juice is processed, the apple can be soaked with a certain concentration of $\mathrm{NaCl}$. After a period of soaking, $\mathrm{NaCl}$ can destroy and inhibit the polyphenol oxidase in the apple. On the other hand, the opportunity for apple to contact with the air is less when $\mathrm{NaCl}$ is easily soaked. This is due to the oxygen in the water. The solubility of $\mathrm{NaCl}$ solution is larger than that in $\mathrm{NaCl}$ solution, and the $\mathrm{NaCl}$ solution has a certain protective effect.

\subsection{The citric acid browning inhibitor.}

During the processing of fruit juice, the browning enzyme of Apple has the best range of $\mathrm{pH}$ value in browning, so it can inhibit the browning of Apple by changing the $\mathrm{pH}$ value of Apple tissue. Citric acid has the effect of reducing the $\mathrm{pH}$ value of Apple tissue.

\subsection{CaCl2 browning inhibitor.}

In the process of processing apple juice, browning is mainly due to the oxidation of enzymes, and the use of $\mathrm{CaCl} 2$ can effectively prevent the occurrence of oxidation and weaken the occurrence of browning. In addition to weakening the oxidation reaction, using $\mathrm{CaCl} 2$ browning inhibitor, the $\mathrm{Ca}$ ion component in the solution can also react with pectin on the apple cell wall and produce pectin calcium, which can increase the hardness of the cell tissue, obstruct the solution in the juice and the contact of the cell tissue liquid and reduce the browning degree.

\subsection{The inhibitory effect of hot blanching on Browning.}

When the apple browning, the optimum reaction temperature of the oxidase is $20 \mathrm{C}$. At 90 , more than $95 \%$ of the enzyme can be inactive. Therefore, the activity of polyphenol oxidase can be lost by high temperature treatment, thus inhibiting the occurrence of browning. However, the time of 
high temperature treatment should not be too long, because the long time high temperature treatment will soften the tissue of the juice, and affect the taste of the juice. The best treatment time can be obtained through repeated tests, and the processing quality of the juice can be guaranteed.

\section{Study on the effect of 3 apple juice browning inhibition test}

In order to study the effect of various inhibitors on the inhibition effect of apple juice processing, different concentrations of Sodium Chloride Solution, calcium chloride solution and citric acid solution were made, and the effects of different high temperatures on Browning were tested. The best inhibition effect could be obtained by comparing the results of the test. Taking Sodium Chloride Solution as an example, as shown in Table 1, the solution with concentration of $1.25 \%$, $1.5 \%, 1.75 \%, 2 \%$ and $2.25 \%$, respectively, was tested.

Table 1 The test with different concentrations of $\mathrm{NaCl}$ solution

\begin{tabular}{|l|l|}
\hline Concentration of NaCl solution & A value (absorbance value) \\
\hline 1.25 & 0.022 \\
\hline 1.5 & 0.018 \\
\hline 1.75 & 0.016 \\
\hline 2.0 & 0.025 \\
\hline 2.25 & 0.032 \\
\hline
\end{tabular}

The activity of polyphenol oxidase in apple after treatment with different concentrations of sodium chloride was also different. As shown in Figure 1, with the increase of Sodium Chloride Solution concentration, the activity of the enzyme first decreased and then increased. At the concentration of $1.5 \%$, the activity of the enzyme was the least. At this time, the inhibition effect was the best and the possibility of browning was the least.

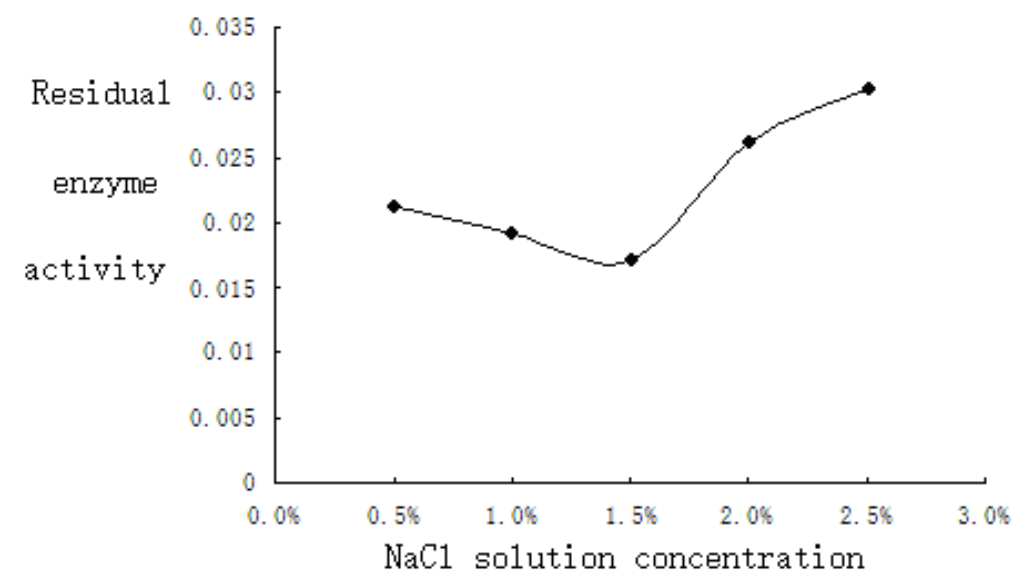

Fig.1 The effect of $\mathrm{NaCl}$ solution with different concentrations on Browning

In order to study the effects of various inhibitors, the effects of several other inhibitors and high temperature treatment were compared, and the optimum mixture ratio of several inhibitors was obtained by orthogonal test. The experimental results showed that $1.5 \%$ of sodium chloride, $0.80 \%$ citric acid, $0.60 \%$ calcium chloride and $0.35 \%$ ascorbic acid were used. When mixed, the effect of the inhibitor is the best, and the effect of the retest reagents is better than that of the high temperature hot blanching and the reagents.

\section{Conclusion}

The causes of browning in the processing of apple juice and the inhibition technology were studied. The different inhibitors were studied. The optimum concentration ratio of several inhibitors was obtained by testing different concentrations of inhibitors. Finally, the mixture of different 
concentration ratio solution was mixed. A better compound inhibitor was compared with physical treatment method to verify the superiority of the inhibition effect. Fresh apple juice is a kind of all natural fruit juice. It is a kind of all natural fruit juice. Based on its freshness and nutrition, fresh apple juice will be more and more popular with consumers.

\section{References}

[1] Li Peiyan, xenophobia. A comparative study of browning in the process of processing red Fuji, Qin Guan and Australian green apple juice [J]. Journal of Shaanxi Normal University, 2008, 36 (6): 96-100.

[2] Hao Huiying, Zhao Guang chela. Polyphenols in apple wine and browning [J]. liquor making, 2002, 3:63-65.

[3] Lin Xiaolan, Chen Jin Quan. Enzymatic browning factors in the processing of Castanea henryi and their control [J]. Journal of Fujian Kasetsart University, 2001, 30 (2): 240-243.

[4] Fan Minghui, Wang Miao, Yin Shaoqian, Gu Guo Yuan. The study of the pre browning of apple juice and the partial characteristics of apple PPO [J]. Food and fermentation industry, 2005, 33-36.

[5] Xu Jing, Han Jianchun. The optimization of the fermentation conditions of the high yield strain of elastase and the preliminary study on the properties of elastase [J]. Food industry technology, 2008.26 (6): 142-144.

[6] Hao Jianguo, Xue Yanfen, Ma Yan He. Isolation, identification and enzymatic characteristics of a protease producing alkalophilic strain [J]. Acta microbiology Sinica, 2010, 50 (1): 54-59.

[7] Huang Xiao Jing, Wu Jihong, Chen Fang, et al. Selection of fruit maturity in cloudy juice processing of apple.2005, 31 (2): 10-13.

[8] Lin Xiangdong, Zhang Qi, Li Jixin. Study on Polyphenol Oxidase characteristics and browning prevention measures in low confectionery sauce. Food science and technology, 2002, (7): 35-37.

[9] Zhao Guangyuan, Wang Zhang, Xu Shi Ying. Effects of steam heat treatment on the color and turbidity stability of cloudy apple juice during crushing. Food and fermentation industry, 2004, (10): 26-30.

[10] Yang Wei, Liu Jing, Lv Chunjing, Wang Chieh, Zhang Sumin. The effects of calcium chloride and ascorbic acid treatment on the quality and browning of fresh cut apples [J] Chinese agricultural science 2010,43 (16): 3402-3410.

[11] Han Tao, Li Liping, Zhao Jia. Color change and color protection technology of cut yam slices during storage period [J]. Food industry science and technology, 2005, 26 (1): 175-177.

[12] Fu Yu Cheng, Wang Niya, Du Jinhua. Extraction and partial enzymatic characteristics of polyphenol oxidase from apple of Jin Shuai [J]. [J]. food industry technology, 2006, 2 (27): 59-62.

[13] Liu Xinghua, Ma Hongjuan, Fuji apple flesh browning on the protection of enzyme activity and membrane peroxidation, Northwest Agricultural Journal, 2004, 13 (1): 80-83. 